\title{
(C) OPEN ACCESS \\ Pain management after laparoscopic hysterectomy: systematic review of literature and PROSPECT recommendations
}

\author{
Philipp Lirk, ${ }^{1}$ Juliette Thiry, ${ }^{2}$ Marie-Pierre Bonnet, ${ }^{3}$ Girish P Joshi, ${ }^{4}$ Francis Bonnet, ${ }^{2}$ for \\ the PROSPECT Working Group
}

'Department of Anesthesiology, Brigham and Women's Hospital, Harvard Medical School, Boston, Massachusetts, USA

${ }^{2}$ Department of Anesthesiology and Intensive Care Hôpital Tenon, Groupe Hospitalier Universitaire Est Parisien, Assistance Publique Hôpitaux de Paris, Université Pierre \& Marie, Paris, France ${ }^{3}$ Department of Anesthesiology and Intensive Care Hôpital Cochin, Assistance Publique Hôpitaux de Paris, Université René Descartes, Paris, France ${ }^{4}$ Department of Anesthesiology and Pain Management, University of Texas Southwestern Medical Center at Dallas, Dallas, Texas, USA

\section{Correspondence to} Dr Philipp Lirk, Department of Anesthesiology, Perioperative and Pain Medicine, Brigham and Women's Hospital, Harvard Medical School, Boston, MA 02115, USA;

plirk@bwh.harvard.edu

Interim data from this analysis were presented at the 36th Annual Meeting of the European Society of Regional Anaesthesia and Pain Therapy (ESRA), Lugano, Switzerland, September 2017.

Received 10 August 2018 Revised 23 December 2018 Accepted 31 December 2018 Published Online First 3 February 2019

\section{ABSTRACT \\ Background and objectives Laparoscopic} hysterectomy is increasingly performed because it is associated with less postoperative pain and earlier recovery as compared with open abdominal hysterectomy. The aim of this systematic review was to evaluate the available literature regarding the management of pain after laparoscopic hysterectomy.

Strategy and selection criteria Randomized controlled trials evaluating postoperative pain after laparoscopic hysterectomy published between January 1996 and May 2018 were retrieved, according to the Preferred Reporting Items for Systematic Reviews and Meta-Analyses guidelines, from the EMBASE and MEDLINE databases and the Cochrane Register of Controlled Trials. Efficacy and adverse effects of analgesic techniques were assessed.

Results Of the 281 studies initially identified, 56 were included. Of these, 31 assessed analgesic or anesthetic interventions and 25 assessed surgical interventions. Acetaminophen, non-steroidal anti-inflammatory drugs, and dexamethasone reduced opioid consumption. Limited evidence hindered recommendations on alpha-2-agonists. Inconsistent evidence was found in the studies investigating pregabalin and transversus abdominis plane block, and no evidence was found for intraperitoneal local anesthetics, port site infiltration, or single-port laparoscopy. Measures to lower peritoneal insufflation pressure or humidify or heat insufflated gas seem to reduce the incidence of shoulder pain, but not abdominal pain.

Conclusions The baseline analgesic regimen for laparoscopic hysterectomy should include acetaminophen, a non-steroidal anti-inflammatory drug, dexamethasone, and opioids as rescue analgesics.

\section{INTRODUCTION}

Hysterectomy is one of the most common major surgical procedures performed in women. The laparoscopic approach is increasingly used, as it is associated with reduced postoperative pain and morbidity, as well as earlier recovery and a shorter hospital stay when compared with open hysterectomy. ${ }^{1-5}$ However, pain may still be quite severe, particularly in the early postoperative period. ${ }^{67}$ Literature assessing non-opioid pain therapy for patients undergoing vaginal and laparoscopic hysterectomy has been recently evaluated. ${ }^{8}$ However, this study was not truly procedure-specific, including both vaginal and laparoscopic hysterectomies, and did not focus on opioid analgesics, anesthetics, and surgical technique.

The PROSPECT (PROcedure-SPECific postoperative pain managemenT) Working Group is a collaboration of anesthesiologists and surgeons which formulates evidence-based recommendations for postoperative pain management that are specific for different surgical procedures. ${ }^{9}{ }^{10}$ In addition to procedure-specific evidence, clinical practice information is used to provide overall recommendations considering the efficacy and adverse effects of an analgesic technique (www.postoppain.org).

The aim of this systematic review was to evaluate the available literature on the management of pain after laparoscopic hysterectomy. Postoperative pain outcomes (pain scores and analgesic requirements) were the primary focus, but other recovery outcomes, including adverse effects, were also assessed, when reported, and the limitations of the data were reviewed. The ultimate aim was to use the available evidence to develop recommendations for pain management in this patient population.

\section{METHODS}

A systematic review of randomized controlled trials (RCTs) published between January 1996 and May 2018 assessing analgesic interventions for laparoscopic hysterectomy was performed according to the Preferred Reporting Items for Systematic Reviews and Meta-Analyses guidelines using EMBASE, PubMed, and the Cochrane Register of Controlled Trials. ${ }^{11}$ The search terms related to pain interventions for laparoscopic hysterectomy surgery without language restriction included "laparoscopic hysterectomy" AND ("postoperative pain” OR “analgesia” OR "visual analog score” OR "local anesthetic" OR "regional anesthesia" OR "regional analgesia" OR "infiltration" OR “TAP block" OR "nonsteroidal antiinflammatory drugs" OR “non opioid analgesic" OR “opioid” OR “dexamethasone” OR “gabapentin” OR “pregabalin” OR "ketamine" OR "paracetamol” OR "acetaminophen" OR “corticosteroids"). We also manually retrieved publications referred in studies identified by our preceding search.

\section{Study inclusion/exclusion criteria}

We included RCTs assessing analgesic, anesthetic, or surgical interventions for laparoscopic hysterectomy with pain intensity measured by Visual Analog 
Scale (VAS) or Numerical Rating Scale (NRS). Studies that did not measure pain intensity and studies including laparoscopic hysterectomy that reported data pooled with other surgical procedures were excluded. Three authors (JT, PL, and M-PB) conducted the literature search, while four authors (JT, M-PB, $\mathrm{PL}$, and FB) were involved in the review of the literature search and exclusion of irrelevant articles where ambiguous. The results of the literature search were grouped according to analgesic modality and presented to the entire Working Group (see list of Authors) in two subsequent meetings half a year apart. The authors proposed interpretations of the findings, and the entire group was engaged in the drafting of the final recommendations in several iterations, collating rounds of individual comments on the evidence and draft recommendations, followed by roundtable discussions and further rounds of individual comments. When consensus had been obtained, the lead authors compiled the manuscript, and again all the Working Group members participated actively in the writing and discussion. The methodology of the PROSPECT group is unique in that it aims to synthesize clinical evidence while considering the risks and benefits of interventions as well as taking into account study design. Specifically, the group seeks to determine the relevance of study interventions in current perioperative care practice and critically evaluate the baseline pain treatment. Recommendations are given when at least two congruent studies support an intervention.

\section{Quality of included studies}

The following were the criteria used to assess the quality of eligible studies: statistical analyses and patient follow-up assessment-indication whether statistical analysis was reported and whether follow-up was more or less than $80 \%$ of the patients; and allocation concealment assessment-indication whether there was adequate guarantee of blinding for treatment assignment (A, adequate; B, unclear; C, inadequate; D, not used; tables 1 and 2). ${ }^{12}$ Quality of studies was graded using the
Jadad Numerical Score (total 1-5) using the criteria based on appropriate randomization, double-blinding, and statements of possible withdrawals. ${ }^{13}$

\section{Analysis of outcome and statistical analysis}

We focused on pain intensity whenever it was the primary or secondary outcome. Unless specified otherwise, it was assumed that the pain scores were assessed at rest. We also retrieved comparative opioid consumption, particularly when the study design included patient-controlled intravenous opioid (morphine or morphine equivalent) administration. The studies were stratified according to the intervention evaluated: analgesic, anesthetic, or surgical. The effectiveness of each intervention was evaluated qualitatively by assessing the number of studies showing a significant difference. For the purpose of this review, we define a change $>10 \mathrm{~mm}$ on a $100 \mathrm{~mm}$ VAS as clinically important. ${ }^{14}$

\section{RESULTS}

A total of 281 studies assessing analgesic interventions for laparoscopic hysterectomy were identified (figure 1). Of these, 23 retrospective studies, 31 non-randomized prospective, and 153 other publications were excluded. Of the remaining 74 RCTs, 18 studies were excluded ( 9 studies did not provide reproducible evaluation of pain, 2 studies expressed the results in figures and data could not be validated, and 7 studies did not provide data specifically for laparoscopic hysterectomy as data were pooled with other surgical procedures). Thus, 56 RCTs were included, 31 concerning analgesic or anesthetic treatments, and 25 concerning surgical procedures (figure 1 ).

The quality scores of all the studies reviewed (allocation concealment, Jadad score, and level of evidence) are reported in table 2, while details on analgesic interventions are given in table 3. For the qualitative analysis, the included trials were

Table 1 Relationship between study quality and source of evidence, LoE, and grades of recommendation

\begin{tabular}{|c|c|c|c|c|c|c|}
\hline \multirow[b]{2}{*}{ Study type } & \multicolumn{4}{|l|}{ Study quality assessments } & \multirow[b]{2}{*}{ LoE } & \multirow[b]{2}{*}{$\begin{array}{l}\text { Grade of recommendation } \\
\text { (based on overall LoE, } \\
\text { considering balance of } \\
\text { clinical practice information } \\
\text { and evidence) }\end{array}$} \\
\hline & $\begin{array}{l}\text { Statistical analyses } \\
\text { and patient follow- } \\
\text { up assessment }\end{array}$ & $\begin{array}{l}\text { Allocation } \\
\text { concealment* }\end{array}$ & Jadad scores & $\begin{array}{l}\text { Additional assessment } \\
\text { of overall study } \\
\text { quality required to } \\
\text { judge LoE }\end{array}$ & & \\
\hline $\begin{array}{l}\text { Systematic review with } \\
\text { homogeneous results }\end{array}$ & NA & NA & NA & NA & 1 & A \\
\hline \multirow[t]{4}{*}{ RCT } & \multirow[t]{4}{*}{$\begin{array}{l}\text { Statistics reported and AND } \\
>80 \% \text { follow-up }\end{array}$} & $\begin{array}{l}A \\
O R\end{array}$ & $(1-5)$ & NA & \multirow[t]{4}{*}{1} & \multirow{4}{*}{$\begin{array}{l}\text { A } \\
\text { (based on two or more studies } \\
\text { or a single, large, well-designed } \\
\text { study) }\end{array}$} \\
\hline & & B & $(3-5)$ & NA & & \\
\hline & & OR & & & & \\
\hline & & B & $(1-2)$ & Yes & & \\
\hline \multirow[t]{5}{*}{ RCT } & \multirow{5}{*}{$\begin{array}{l}\text { Statistics not reported AND/OR } \\
\text { or questionable or } \\
<80 \% \text { follow-up }\end{array}$} & B & $(1-2)$ & Yes & \multirow[t]{5}{*}{2} & \multirow{5}{*}{$\begin{array}{l}\text { B } \\
\text { (or extrapolation from one } \\
\text { procedure-specific } \\
\text { LoE } 1 \text { study) }\end{array}$} \\
\hline & & OR & & & & \\
\hline & & c & $(1-5)$ & NA & & \\
\hline & & $\mathrm{OR}$ & & & & \\
\hline & & D & $1-5)$ & NA & & \\
\hline $\begin{array}{l}\text { Non-systematic review, cohort } \\
\text { study, case study (eg, adverse } \\
\text { effects) }\end{array}$ & NA & NA & & & 3 & C \\
\hline $\begin{array}{l}\text { Clinical practice information } \\
\text { (expert opinion); inconsistent } \\
\text { evidence }\end{array}$ & NA & NA & & & 4 & D \\
\hline
\end{tabular}

*Allocation concealment is given as $A$ adequate, $B$ unclear, $C$ inadequate, and $D$ not used.

LoE, level of evidence; NA, not applicable; RCT, randomized controlled trial. 
Table 2 Quality assessment and level of evidence assigned to the randomized trials included in this review

\begin{tabular}{|c|c|c|c|c|c|}
\hline $\begin{array}{l}\text { Study } \\
\text { (reference } \\
\text { number) }\end{array}$ & $\begin{array}{l}\text { Statistics } \\
\text { reported }\end{array}$ & Follow-up $>80 \%$ & $\begin{array}{l}\text { Allocation } \\
\text { concealment }\end{array}$ & $\begin{array}{l}\text { Jadad } \\
\text { score }\end{array}$ & $\begin{array}{l}\text { Level of } \\
\text { evidence }\end{array}$ \\
\hline Jokela et $a l^{15}$ & $Y$ & $Y$ & A & 5 & 1 \\
\hline Kim et $a l^{16}$ & $Y$ & Y & A & 4 & 1 \\
\hline Kim et $a l^{17}$ & $Y$ & $Y$ & B & 4 & 1 \\
\hline Moon et $a l^{18}$ & $Y$ & $Y$ & A & 5 & 1 \\
\hline Jokela et $a l^{19}$ & $Y$ & $Y$ & A & 5 & 1 \\
\hline $\begin{array}{l}\text { Thangaswamy } \\
\text { et }\left.a\right|^{20}\end{array}$ & $Y$ & $Y$ & A & 5 & 1 \\
\hline $\begin{array}{l}\text { Nam and } \\
\text { Yoon }\end{array}$ & $Y$ & Y & B & 1 & 2 \\
\hline Jokela et $a^{22}$ & $Y$ & $\mathrm{Y}$ & A & 5 & 1 \\
\hline Asgari et a $\left.\right|^{23}$ & $Y$ & $Y$ & A & 5 & 1 \\
\hline Kim et $a l^{24}$ & $Y$ & $Y$ & A & 5 & 1 \\
\hline Jung et $a l^{25}$ & $Y$ & $Y$ & A & 4 & 1 \\
\hline Choi et $a l^{26}$ & $Y$ & $Y$ & A & 4 & 1 \\
\hline Lenz et $a l^{27}$ & $Y$ & $Y$ & A & 4 & 1 \\
\hline Kim et $\left.a\right|^{28}$ & $Y$ & $Y$ & A & 3 & 1 \\
\hline Kim et $a l^{29}$ & $Y$ & $Y$ & A & 5 & 1 \\
\hline Park et $\left.a\right|^{30}$ & $Y$ & $Y$ & A & 4 & 1 \\
\hline Lee et $a \beta^{31}$ & $Y$ & $Y$ & B & 3 & 1 \\
\hline Lee et $a^{\beta 2}$ & $Y$ & $Y$ & A & 5 & 1 \\
\hline $\begin{array}{l}\text { Hong and } \\
\text { Lim }^{33}\end{array}$ & Y & $Y$ & A & 5 & 1 \\
\hline $\begin{array}{l}\text { De Oliveira } \\
\text { et } a^{34}\end{array}$ & $Y$ & $Y$ & A & 4 & 1 \\
\hline Kane et $a l^{35}$ & Y & Y & A & 4 & 1 \\
\hline Calle et $\left.a\right|^{36}$ & $Y$ & $Y$ & A & 5 & 1 \\
\hline $\begin{array}{l}\text { El Hachem } \\
\text { et } a l^{37}\end{array}$ & $Y$ & Y & A & 5 & 1 \\
\hline Torup et $\left.a\right|^{\beta 8}$ & $Y$ & $Y$ & A & 5 & 1 \\
\hline $\begin{array}{l}\text { Hutchins et } \\
a^{\beta 39}\end{array}$ & $Y$ & $Y$ & A & 5 & 1 \\
\hline Ghisi et $a l^{40}$ & $Y$ & $Y$ & B & 3 & 1 \\
\hline $\begin{array}{l}\text { Guardabassi } \\
\text { et } a l^{41}\end{array}$ & $Y$ & $Y$ & B & 3 & 1 \\
\hline Arden et $a l^{42}$ & $Y$ & $Y$ & A & 5 & 1 \\
\hline $\begin{array}{l}\text { Andrews et } \\
a l^{43}\end{array}$ & Y & $Y$ & A & 5 & 1 \\
\hline Barron et a $\left.\right|^{44}$ & $Y$ & $Y$ & A & 5 & 1 \\
\hline Nelskyla et $a{ }^{45}$ & $Y$ & $Y$ & A & 2 & 1 \\
\hline $\begin{array}{l}\text { Pokkinen et } \\
a{ }^{46}\end{array}$ & $Y$ & $Y$ & B & 3 & 1 \\
\hline
\end{tabular}

\begin{tabular}{|c|c|c|c|c|c|}
\hline Kim et $\left.a\right|^{47}$ & Y & $Y$ & A & 4 & 1 \\
\hline Muzii et al ${ }^{48}$ & Y & $Y$ & A & 3 & 1 \\
\hline Song et $a l^{49}$ & Y & $Y$ & A & 5 & 1 \\
\hline $\begin{array}{l}\text { Eggemann } \\
\text { et }\left.a\right|^{50}\end{array}$ & Y & $Y$ & A & 5 & 1 \\
\hline Chen et $a l^{51}$ & Y & $Y$ & A & 3 & 1 \\
\hline Li et $a F^{52}$ & Y & $Y$ & A & 2 & 1 \\
\hline Jung et $a l^{53}$ & Y & $Y$ & A & 2 & 1 \\
\hline Chung et $a l^{54}$ & Y & $Y$ & D & 3 & 2 \\
\hline Kim et $a P^{55}$ & $Y$ & $Y$ & $D$ & 3 & 2 \\
\hline Song et $\left.a\right|^{56}$ & Y & $Y$ & D & 3 & 2 \\
\hline Fanfani et $\left.a\right|^{57}$ & Y & $Y$ & B & 3 & 2 \\
\hline Paraiso et a $\left.\right|^{58}$ & Y & $Y$ & A & 5 & 1 \\
\hline Sarlos et $\left.a\right|^{59}$ & Y & $Y$ & A & 3 & 1 \\
\hline
\end{tabular}

Table 2 Continued

\begin{tabular}{|c|c|c|c|c|c|}
\hline $\begin{array}{l}\text { Study } \\
\text { (reference } \\
\text { number) }\end{array}$ & $\begin{array}{l}\text { Statistics } \\
\text { reported }\end{array}$ & Follow-up $>80 \%$ & $\begin{array}{l}\text { Allocation } \\
\text { concealment }\end{array}$ & $\begin{array}{l}\text { Jadad } \\
\text { score }\end{array}$ & $\begin{array}{l}\text { Level of } \\
\text { evidence }\end{array}$ \\
\hline $\begin{array}{l}\text { Tchartchian } \\
\text { et a/ }\end{array}$ & $Y$ & $\mathrm{~N}$ & B & 1 & 2 \\
\hline Ghezzi et $a^{61}$ & Y & $Y$ & A & 5 & 1 \\
\hline Acton et $a^{/ 62}$ & Y & $Y$ & D & 3 & 2 \\
\hline Shen et $a^{63}$ & Y & Y & A & 3 & 1 \\
\hline Bogani et $a^{64}$ & Y & Y & A & 5 & 1 \\
\hline Madsen et $a^{65}$ & Y & Y & A & 5 & 1 \\
\hline $\begin{array}{l}\text { Herrmann and } \\
\text { De Wilde }^{66}\end{array}$ & Y & Y & A & 5 & 1 \\
\hline Radosa et a ${ }^{67}$ & Y & Y & B & 3 & 2 \\
\hline Roy et $a^{68}$ & Y & Y & A & 2 & 1 \\
\hline Fagotti et $a^{69}$ & Y & Y & A & 5 & 1 \\
\hline $\begin{array}{l}\text { Rothmund et } \\
a l^{70}\end{array}$ & Y & Y & B & 2 & 2 \\
\hline
\end{tabular}

Statistics reported andFollow-up $>80 \%$ information is given as $\mathrm{Y}$ for Yes and $\mathrm{N}$ for No. Allocation concealment is given as $A$ adequate, $B$ unclear, $C$ inadequateand $D$ not used. Jadad score is numerical from 1-5.

divided into three groups-analgesic interventions, anesthetic interventions, and surgical procedures.

\section{Analgesic interventions}

Systemic non-opioid analgesics

Acetaminophen and non-steroidal anti-inflammatory drugs

In a three-arm study, the analgesic effects of acetaminophen/ ondansetron, acetaminophen/placebo, or placebo/placebo were investigated. The cumulative 24-hour dose of the rescue analgesic, oxycodone, was lower in patients receiving acetaminophen, but not influenced by ondansetron. Pain scores and opioid side effects were not different between groups. ${ }^{15}$ The efficacy of bupivacaine port site infiltration and ketorolac administration was compared in a four-arm study, where the lowest pain scores during the first 6 hours following surgery were found in the group which received both port site infiltration and ketorolac versus placebo. ${ }^{16}$ Further, another study compared intravenous patient-controlled analgesia (PCA) using remifentanil, two combinations of remifentanil and ketorolac, and fentanyl combined with ketorolac. Ketorolac had a sparing effect on remifentanil consumption and decreased sedation score by, on average, approximately 0.5 points. ${ }^{17}$

Moon and colleagues ${ }^{18}$ investigated the opioid-sparing effects of either $200 \mathrm{mg}$ or $400 \mathrm{mg}$ nefopam when added to a fentanyl intravenous PCA over the first 48 postoperative hours. Cumulative on-demand fentanyl consumption during the first 48 hours postoperatively was $236 \pm 128 \mu \mathrm{g}$ vs $107 \pm 105 \mu \mathrm{g}$ for the fentanyl-only versus the fentanyl-nefopam group, while pain scores, side effects, and patient satisfaction were not different. ${ }^{18}$

In summary, administration of non-opioid drugs such as acetaminophen, ketorolac, or nefopam has an opioid-sparing effect, but effects on mobilization and opioid-related side effects are unclear. Even though not powered to detect such an outcome variation, neither of the studies using non-opioids reported excessive bleeding in the non-steroidal anti-inflammatory drug (NSAID) groups.

\section{Steroids}

The potential analgesic efficacy of dexamethasone was tested in a four-arm study comparing three doses of dexamethasone 
Primary search results: $\mathbf{2 8 1}$

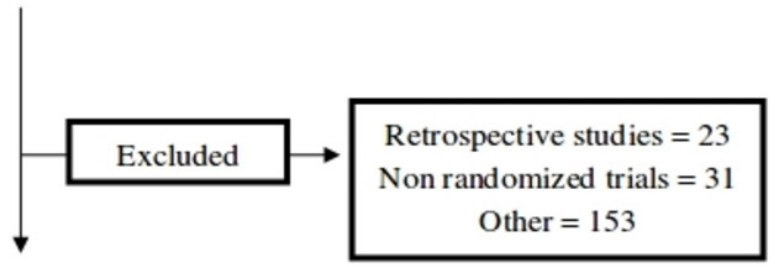

Studies considered for inclusion $=\mathbf{7 4}$

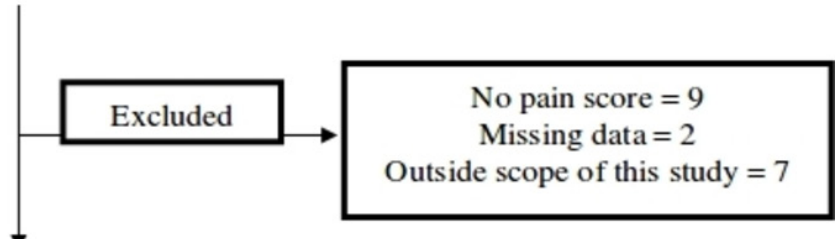

Studies included in qualitative analysis: $\mathbf{5 6}$

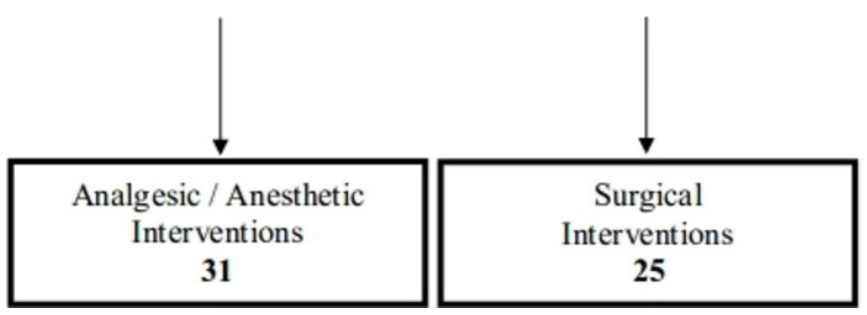

Figure 1 PRISMA flow chart of literature search. PRISMA, Preferred Reporting Items for Systematic Reviews and Meta-Analyses.

(15 mg, $10 \mathrm{mg}$, and $5 \mathrm{mg}$, intravenously) with placebo, administered before induction of anesthesia. ${ }^{19}$ During the first 24 hours postoperatively, cumulative oxycodone dose was lower in the $15 \mathrm{mg}$ dexamethasone $(0.34$ [range $0.11-0.87$ ] $\mathrm{mg} / \mathrm{kg})$ group as compared with placebo $(0.55$ [range $0.19-1.13] \mathrm{mg} / \mathrm{kg})$, and during the first 2 hours postoperatively oxycodone consumption in the $10 \mathrm{mg}$ group was also lower. At the same time, resting and dynamic VAS scores as well as side effects (including sedation) were comparable. ${ }^{19}$ Another study compared dexamethasone $4 \mathrm{mg}$ or $8 \mathrm{mg}$ administered 2 hours before induction of anesthesia with placebo. ${ }^{20}$ All patients received acetaminophen and ibuprofen as baseline analgesics. Pain scores were not different, but cumulative postoperative fentanyl demand during the first 24 hours was less in the dexamethasone $8 \mathrm{mg}$ group $(547 \pm 69$ $\mu \mathrm{g})$ compared with the placebo $(646 \pm 77 \mu \mathrm{g})$ and dexamethasone $4 \mathrm{mg}$ groups. Postoperative nausea and vomiting (PONV) were significantly less frequent in the dexamethasone $8 \mathrm{mg}$ group. No adverse effects related to dexamethasone were observed. ${ }^{20}$ Further, dexamethasone $10 \mathrm{mg}$ plus ondansetron was compared with ondansetron only, with no differences in the two groups in the mean VAS scores and mean opioid consumption, but the rate of PONV was lower in the group receiving both drugs. ${ }^{21}$

In summary, dexamethasone seems to exhibit dose-dependent opioid-sparing effects and contributes to reducing PONV, but does not reduce pain scores.

\section{Gabapentinoids}

The pre-emptive analgesic effect of two doses of pregabalin (300 $\mathrm{mg}$ and $600 \mathrm{mg}$ ) or diazepam $(10 \mathrm{mg})$ was compared in a three-arm study, with the medications given as premedication and after 12 hours. $^{22}$ Only opioids were administered on the day of surgery. The VAS scores for pain at rest, on movement, and on coughing were comparable in the three study groups. The cumulative dose of oxycodone (0-24 hours after surgery) was lower in the pregabalin $600 \mathrm{mg}$ group as compared with the diazepam group, but patients receiving pregabalin suffered from side effects such as dizziness, blurred vision, and headaches more frequently. Asgari and colleagues ${ }^{23}$ randomized 96 patients to two preoperative and one postoperative dose of pregabalin 75,150 , or $300 \mathrm{mg}$, or placebo $(\mathrm{n}=24 \mathrm{each})$. The authors found that both $150 \mathrm{mg}$ and $300 \mathrm{mg}$ of pregabalin decreased pain scores, but in the $300 \mathrm{mg}$ group the sedation score was significantly higher than in the placebo and the lower dosed groups. ${ }^{23}$ Curiously, not a single patient in the pregabalin $300 \mathrm{mg}$ and the placebo group required any rescue opioids despite the fact that no standardized multimodal regimen was used. In conclusion, pregabalin has potential opioid-sparing effects, but especially at higher doses it may be associated with side effects precluding its widespread use.

\section{Alpha-2 adrenergic agonists}

Kim and colleagues ${ }^{24}$ investigated the effects of different doses of dexmedetomidine on postoperative shivering. In patients receiving $1 \mu \mathrm{g} / \mathrm{kg}$ body weight of dexmedetomidine, time to rescue analgesic was prolonged by, on average, $11 \mathrm{~min}$ as compared with placebo, and the number of patients requiring rescue analgesics in the postanesthesia care unit (PACU) $(93 \%$ of control patients) was reduced to $50 \%$ and $43 \%$ in patients 
Table 3 Summary of key results from studies evaluating systemic analgesics, analgesics adjuncts, regional anesthesia, and surgical procedures in patients undergoing $\mathrm{LH}$

\begin{tabular}{|c|c|c|c|}
\hline Study & Study design/adequate baseline analgesia & Pain scores & Cumulative opioid doses \\
\hline \multicolumn{4}{|l|}{$\begin{array}{l}\text { Baseline analgesia } \\
\text { (systemic non-opioids) }\end{array}$} \\
\hline Jokela et $a l^{15}$ & $\begin{array}{l}\text { Acetaminophen } 1 \text { g/ondansetron every } 6 \text { hours }(n=40) \text {, } \\
\text { acetaminophen/placebo }(n=40) \text { and placebo/placebo }(n=40) \text {. }\end{array}$ & NS & $\begin{array}{l}\text { Rescue opioid oxycodone lower in } \\
\text { acetaminophen group }(0.34 \pm 0.15 \text { vs } \\
0.43 \pm 0.18 \mathrm{mg} / \mathrm{kg}) \text {. }\end{array}$ \\
\hline Kim et $a l^{16}$ & $\begin{array}{l}\text { Ketorolac intramuscularly + bupivacaine infiltration }(n=21) \text {, } \\
\text { ketorolac intramuscularly/placebo }(n=20) \text {, bupivacaine/placebo } \\
(n=21) \text {, placebo/placebo }(n=21) \text {. }\end{array}$ & $\begin{array}{l}\text { Lower in the ketorolac bupivacaine } \\
\text { group by } 1-1.7 \text { NRS points. }\end{array}$ & $\begin{array}{l}\text { Lower in ketorolac bupivacaine group vs } \\
\text { placebo. }\end{array}$ \\
\hline Kim et al ${ }^{17}$ & $\begin{array}{l}\text { Intravenous PCA remifentanil }(n=20) \text {, intravenous PCA } \\
\text { remifentanil + ketorolac ( } 2 \text { doses, } n=19 \text { and } n=20) \text {, intravenous } \\
\text { PCA fentanyl }(n=20) \text {. }\end{array}$ & NS & Lower in patients with ketorolac. \\
\hline Moon et al ${ }^{18}$ & $\begin{array}{l}\text { Fentanyl PCA with nefopam } 2 \text { or } 4 \mathrm{mg}(n=28 \text { and } 26) \text { per PCA } \\
\text { bolus or fentanyl only }(n=27) \text {. }\end{array}$ & NS & Lower in both nefopam groups. \\
\hline \multicolumn{4}{|l|}{ Analgesic adjuncts } \\
\hline Jokela et al ${ }^{19}$ & $\begin{array}{l}3 \text { intravenous doses of dexamethasone } 15 \mathrm{mg}, 10 \mathrm{mg} \text {, and } 5 \text { mg, } \\
30 \text { patients in each group. } \\
\text { Baseline analgesia: acetaminophen and ibuprofen from POD } 1 .\end{array}$ & NS & $\begin{array}{l}\text { Lower in dexamethasone } 10 \mathrm{mg} \text { and } 15 \\
\text { mg doses. }\end{array}$ \\
\hline Thangaswamy et al $\left.\right|^{20}$ & $\begin{array}{l}\text { Intravenous } 4 \mathrm{mg}(\mathrm{n}=18) \text { and } 8 \mathrm{mg}(\mathrm{n}=19) \text { dexamethasone vs } \\
\text { placebo }(\mathrm{n}=18) \text {. } \\
\text { Baseline analgesia: acetaminophen and ibuprofen after discharge } \\
\text { to ward. }\end{array}$ & NS & $\begin{array}{l}\text { Lower for the } 8 \mathrm{mg} \text { dexamethasone dose } \\
\text { vs placebo. }\end{array}$ \\
\hline Nam et $a l^{21}$ & $\begin{array}{l}\text { Dexamethasone } 10 \mathrm{mg}+\text { ondansetron }(\mathrm{n}=25) \text { vs ondansetron } \\
(\mathrm{n}=25) \text {. } \\
\text { Baseline analgesia: not reported. }\end{array}$ & NS & NS \\
\hline Jokela et $a l^{22}$ & $\begin{array}{l}2 \text { doses of pregabalin ( } 300 \mathrm{mg}, \mathrm{n}=31 ; 600 \mathrm{mg}, \mathrm{n}=30 \text { ) vs } \\
\text { diazepam }(\mathrm{n}=30) \text {. } \\
\text { Baseline analgesia: acetaminophen and ibuprofen from POD } 1 .\end{array}$ & NS & $\begin{array}{l}\text { Oxycodone rescue lower for the } 600 \mathrm{mg} \\
\text { pregabalin dose }(0.09 \pm 0.07 \mathrm{mg} / \mathrm{kg}) \mathrm{vs} \\
\text { diazepam }(0.15 \pm 0.13 \mathrm{mg} / \mathrm{kg}) \text {. }\end{array}$ \\
\hline Asgari et $a l^{23}$ & $\begin{array}{l}3 \text { doses of pregabalin }(75,150, \text { or } 300 \mathrm{mg}, \mathrm{n}=24 \text { each). } \\
\text { Baseline analgesia: diclofenac or pethidine on request. }\end{array}$ & $\begin{array}{l}\text { Lower in the } 150 \text { and } 300 \text { mg groups by } \\
\text { between } 1.5 \text { and } 3 \text { NRS points. }\end{array}$ & $\begin{array}{l}\text { No patient in placebo or } 300 \text { mg group } \\
\text { received rescue opioids. }\end{array}$ \\
\hline Kim et $a l^{24}$ & $\begin{array}{l}\text { Bolus dexmedetomidine at end of surgery: placebo, } 0.5,0.75 \text {, or } \\
1 \mu \mathrm{g} / \mathrm{kg} \text { ( } \mathrm{n}=30 \text { each). } \\
\text { Baseline analgesia: Not reported. }\end{array}$ & & $\begin{array}{l}\text { Less likely to require rescue analgesic }(0.75 \\
\text { and } 1 \mu \mathrm{g} / \mathrm{kg} \text { groups). }\end{array}$ \\
\hline Jung et $a l^{25}$ & $\begin{array}{l}\text { Continuous intravenous infusion of dexmedetomidine }(n=25) \text { vs } \\
\text { remifentanil }(n=25) \text {. } \\
\text { Baseline analgesia: ketorolac. }\end{array}$ & NS & \\
\hline Choi et $a l^{26}$ & $\begin{array}{l}\text { Brief intravenous infusion of dexmedetomidine }(n=30) \text { vs } \\
\text { remifentanil }(n=25) \text { vs fentanyl }(n=30) \text {. } \\
\text { Baseline analgesia: ketorolac. }\end{array}$ & NS & \\
\hline \multicolumn{4}{|l|}{ Regional anesthesia } \\
\hline Hong and $\operatorname{Lim}^{33}$ & $\begin{array}{l}\text { Epidural analgesia started before incision ( } n=25 \text {, pre-emptive) vs } \\
\text { epidural started after surgery }(n=25) \text {. } \\
\text { Baseline analgesia: no. }\end{array}$ & $\begin{array}{l}\text { Lower in pre-emptive epidural group by } \\
2-3 \text { NRS points between } 3 \text { and } 12 \text { hours } \\
\text { postoperatively. }\end{array}$ & Lower in pre-emptive epidural group. \\
\hline De Oliveira et $a l^{34}$ & $\begin{array}{l}\text { Preincisional } 20 \mathrm{~mL} \text { infiltration TAP block with ropivacaine }(0.5 \% \text {, } \\
\mathrm{n}=22 ; \text { and } 0.25 \%, \mathrm{n}=21) \text { vs saline }(\mathrm{n}=23) \text {. } \\
\text { Baseline analgesia: acetaminophen, ketorolac, ibuprofen. }\end{array}$ & $\begin{array}{l}\text { Lower in ropivacaine groups by } 1-3 \text { NRS } \\
\text { points on the first POD. }\end{array}$ & Lower in ropivacaine 5 mg/mL group. \\
\hline Kane SM, $2012^{35}$ & $\begin{array}{l}\text { Postoperative } 20 \mathrm{~mL} \text { infiltration TAP block with } 0.5 \% \text { ropivacaine } \\
\text { ( } n=28 \text { ) vs no block }(n=29) \text {. } \\
\text { Baseline analgesia: ketorolac single shot at end of surgery. }\end{array}$ & NS & NS \\
\hline Calle et $a l^{36}$ & $\begin{array}{l}\text { Postoperative } 40 \mathrm{~mL} \text { infiltration TAP block with } 1.5 \mathrm{mg} / \mathrm{kg} \text { body } \\
\text { weight bupivacaine }(\mathrm{n}=100) \text { vs placebo block }(\mathrm{n}=97) \text {. } \\
\text { Baseline analgesia: acetaminophen, ketorolac. }\end{array}$ & $\begin{array}{l}\text { Lower at discharge in TAP group (less } \\
\text { than } 1 \text { NRS point). }\end{array}$ & NS \\
\hline El Hachem et $a \beta^{37}$ & $\begin{array}{l}\text { Postoperative } 30 \mathrm{~mL} \text { ultrasound-guided or laparoscopically } \\
\text { guided TAP block using } 0.25 \% \mathrm{mg} / \mathrm{mL} \text { bupivacaine vs trocar } \\
\text { infiltration ( } \mathrm{n}=88 \text {, each patient as own control). } \\
\text { Baseline analgesia: acetaminophen, ketorolac. }\end{array}$ & NS & NS \\
\hline Torup et $\left.a\right|^{38}$ & $\begin{array}{l}\text { Preincisional } 40 \mathrm{~mL} \text { ultrasound-guided TAP block using } \\
\text { ropivacaine } 0.5 \%(n=34) \text { vs placebo }(n=31) \text {. } \\
\text { Baseline analgesia: acetaminophen, ketorolac. }\end{array}$ & NS & NS \\
\hline Hutchins et $a l^{39}$ & $\begin{array}{l}\text { Preincisional } 30 \mathrm{~mL} \text { bupivacaine } 0.25 \% \text { subcostal TAP block } \\
(\mathrm{n}=30) \text { vs } 30 \mathrm{~mL} 1.3 \% \text { liposomal bupivacaine }(\mathrm{n}=28) . \\
\text { Baseline analgesia: acetaminophen, ibuprofen. }\end{array}$ & $\begin{array}{l}\text { Worst pain score lower by } 2.5 \text { NRS points } \\
\text { during the first } 24 \text { hours and } 2 \text { NRS } \\
\text { points during } 24-48 \text { hours in liposomal } \\
\text { group. }\end{array}$ & $\begin{array}{l}\text { Lower during the first } 24 \text { hours and until } 48 \\
\text { hours in the liposomal group. }\end{array}$ \\
\hline
\end{tabular}




\section{Original article}

\begin{tabular}{|c|c|c|c|}
\hline Study & Study design/adequate baseline analgesia & Pain scores & Cumulative opioid doses \\
\hline Ghisi et a ${ }^{40}$ & $\begin{array}{l}\text { Preincisional } 40 \mathrm{~mL} \text { levobupivacaine } 0.375 \% \text { ( } \mathrm{n}=22) \text { vs no block } \\
(\mathrm{n}=22) \text {, combined with systemic analgesia. } \\
\text { Baseline analgesia: opioid only. }\end{array}$ & NS & NS \\
\hline Guardabassi et a $\left.\right|^{41}$ & $\begin{array}{l}\text { Postoperative opioid PCA plus TAP block }(n=20) \text { vs opioid PCA } \\
(n=20) \text {. }\end{array}$ & NS & NS \\
\hline \multicolumn{4}{|l|}{$\begin{array}{l}\text { Intraperitoneal local } \\
\text { anesthetics }\end{array}$} \\
\hline Arden et $a l^{42}$ & $\begin{array}{l}\text { Intraperitoneal bupivacaine infiltration ( } n=67) \text { vs placebo }(n=73) \text {. } \\
\text { Baseline analgesia: ketorolac, acetaminophen. }\end{array}$ & NS & NS \\
\hline Andrews et $\mathrm{al}^{43}$ & $\begin{array}{l}\text { Continuous intraperitoneal bupivacaine infusion }(n=30) \text { vs } \\
\text { placebo }(n=30) \text {. } \\
\text { Baseline analgesia: non-standardized mix of NSAID, } \\
\text { acetaminophen, opioids. }\end{array}$ & NS & NS \\
\hline \multicolumn{4}{|l|}{ Port site infiltration } \\
\hline Kim et $a l^{16}$ & See above. & $\begin{array}{l}\text { Lower in the ketorolac bupivacaine } \\
\text { group by } 1-1.7 \text { NRS points. }\end{array}$ & $\begin{array}{l}\text { Lower in ketorolac bupivacaine group vs } \\
\text { placebo. }\end{array}$ \\
\hline El Hachem et $a l^{37}$ & See above. & NS & NS \\
\hline Barron et a ${ }^{44}$ & $\begin{array}{l}\text { Bupivacaine } 0.25 \% \text { vs liposomal bupivacaine ( } n=30 \text { each). } \\
\text { No standardized multimodal baseline medication. }\end{array}$ & $\begin{array}{l}\text { Worst pain improved in liposomal group } \\
\text { on POD } 2 \text { and } 3 \text { by } 1.5-2 \text { NRS points. }\end{array}$ & NS \\
\hline \multicolumn{4}{|c|}{ Anesthetic techniques } \\
\hline Nelskyla et a ${ }^{45}$ & $\begin{array}{l}\text { Maintenance with isoflurane }(n=30) \text { vs propofol }(n=30) \text {. } \\
\text { Baseline analgesia: ketoprofen. }\end{array}$ & NS & NS \\
\hline Pokkinen et al ${ }^{46}$ & $\begin{array}{l}\text { Maintenance with sevoflurane }(n=74) \text { vs propofol }(n=74) \text {. } \\
\text { Baseline analgesia: acetaminophen. }\end{array}$ & NS & NS \\
\hline Kim et al ${ }^{47}$ & $\begin{array}{l}\text { Single bolus propofol }(0.5 \text { or } 1 \mathrm{mg} / \mathrm{kg} \text { ) or placebo }(\mathrm{n}=33,34 \text {, and } \\
\text { 40). } \\
\text { Baseline analgesia: ketorolac in PCA. }\end{array}$ & NS & NS \\
\hline \multicolumn{4}{|l|}{ Surgical techniques } \\
\hline Muzii et al ${ }^{48}$ & $\begin{array}{l}\text { LAVH ( } n=40) \text { vs MLH ( } n=41) \text {. } \\
\text { Baseline analgesia: not standardized. }\end{array}$ & $\begin{array}{l}\text { Lower in LAVH group by } 1.5-2 \text { NRS } \\
\text { points on POD } 1 \text { and } 2 \text {. }\end{array}$ & \\
\hline Song et a $\left.\right|^{49}$ & $\begin{array}{l}\text { TLH }(n=38) \text { vs LAVH }(n=38) \text {. } \\
\text { Baseline analgesia: not standardized. }\end{array}$ & $\begin{array}{l}\text { Lower in TLH by } 1 \text { NRS point } 18 \text { hours } \\
\text { after surgery. }\end{array}$ & NS \\
\hline Eggemann et $\left.a\right|^{50}$ & $\begin{array}{l}\text { Vaginal hysterectomy vs LAVH, with or without peritoneal closure } \\
(n=47-50 \text { each). }\end{array}$ & $\begin{array}{l}\text { Lower in vaginal hysterectomy by less } \\
\text { than } 1 \text { NRS point. }\end{array}$ & NS \\
\hline Chen et $a l^{51}$ & $\begin{array}{l}\text { Single-port LAVH ( } n=50) \text { vs the conventional multiport LAVH } \\
(n=52) \text {. } \\
\text { Baseline analgesia: intramuscular pain medicine on request. }\end{array}$ & $\begin{array}{l}\text { Lower in the single-port group by } 1-1.5 \\
\text { NRS points at } 24 \text { and } 48 \text { hours. }\end{array}$ & Lower in the single-port group. \\
\hline Li et $a F^{22}$ & $\begin{array}{l}\text { Single-port LAVH }(n=52) \text { vs conventional LAVH }(n=56) \text {. } \\
\text { Baseline analgesia: not reported. }\end{array}$ & NS & \\
\hline Jung et $a f^{53}$ & $\begin{array}{l}\text { Single-port LAVH }(n=30) \text { vs 4-port LAVH }(n=34) \text {. } \\
\text { Baseline analgesia: tamiflumate, intramuscular ketorolac or } \\
\text { meperidine on request. }\end{array}$ & NS & NS \\
\hline Chung et $a f^{54}$ & $\begin{array}{l}\text { Single-port ( } n=30 \text { ) vs conventional laparoscopy }(n=30) \text {. } \\
\text { Baseline analgesia: PCA and tramadol. }\end{array}$ & NS & $\begin{array}{l}\text { NS but higher incidence of rescue } \\
\text { analgesics in the single-port group. }\end{array}$ \\
\hline Kim et $a f^{55}$ & $\begin{array}{l}\text { Single-port ( } n=122) \text { vs conventional laparoscopy ( } n=121) \text {. } \\
\text { Baseline analgesia: not reported. }\end{array}$ & NS & NS \\
\hline Song et $a P^{56}$ & $\begin{array}{l}\text { Single-port }(n=20) \text { vs conventional laparoscopy }(n=21) \text {. } \\
\text { Baseline analgesia: ketorolac in PCA, zaltoprofen orally. }\end{array}$ & NS & NS \\
\hline Fanfani et $a^{57}$ & $\begin{array}{l}\text { Single-port hysterectomy ( } n=34) \text { vs miniport laparoscopy }(n=34) \text {. } \\
\text { Baseline analgesia: intravenous acetaminophen on demand. }\end{array}$ & $\begin{array}{l}\text { Lower in miniport group by } 1-1.5 \text { NRS } \\
\text { points until } 8 \text { hours postoperatively. }\end{array}$ & $\begin{array}{l}\text { Opioids not reported but rescue analgesics } \\
\text { not different. }\end{array}$ \\
\hline Paraiso et $a^{58}$ & $\begin{array}{l}\text { Conventional ( } n=27) \text { vs robotic-assisted ( } n=26) \text { laparoscopy. } \\
\text { Baseline analgesia: not reported. }\end{array}$ & NS & NS \\
\hline Sarlos et a ${ }^{59}$ & $\begin{array}{l}\text { Robotic vs conventional laparoscopic hysterectomy, }(n=95) \text {. } \\
\text { Baseline analgesia: not reported. }\end{array}$ & NS & NS \\
\hline Tchartchian et $a^{100}$ & $\begin{array}{l}\text { Laparoscopic-assisted combined ( } n=14) \text { vs LAV hysterectomy } \\
(n=12) \text {. } \\
\text { Baseline analgesia: detailed scheme including metamizole, opioid } \\
\text { PCA. }\end{array}$ & NS & NS \\
\hline Ghezzi et a ${ }^{61}$ & $\begin{array}{l}\text { MLH }(n=38) \text { vs LH }(n=38) \text {. } \\
\text { Baseline analgesia: ketorolac, acetaminophen. }\end{array}$ & NS & NS \\
\hline Acton et $a l^{62}$ & $\begin{array}{l}\text { Laparoscopy using } 5 \mathrm{~mm}(\mathrm{n}=36) \text { or } 10 \mathrm{~mm}(\mathrm{n}=40) \text { laparoscope. } \\
\text { Baseline analgesia: acetaminophen, celecoxib. }\end{array}$ & $\begin{array}{l}\text { Less pain by approximately } 1 \text { NRS point } \\
\text { in the } 5 \mathrm{~mm} \text { group. }\end{array}$ & \\
\hline
\end{tabular}




\begin{tabular}{|c|c|c|c|}
\hline Study & Study design/adequate baseline analgesia & Pain scores & Cumulative opioid doses \\
\hline Shen et $a l^{63}$ & $\begin{array}{l}\text { Drain }(n=80) \text { vs no drain }(n=84) \text {. } \\
\text { Baseline analgesia: not reported. }\end{array}$ & Lower for shoulder tip pain. & Lower in the drain group. \\
\hline Bogani et $a l^{64}$ & $\begin{array}{l}\text { Low-pressure }(n=20) \text { vs standard-pressure }(n=22) \text { laparoscopy. } \\
\text { Baseline analgesia: acetaminophen, ketorolac on demand. }\end{array}$ & $\begin{array}{l}\text { Abdominal pain similar, shoulder tip pain } \\
\text { lower in low-pressure group. }\end{array}$ & Rescue analgesics NS. \\
\hline Madsen et $a l^{65}$ & $\begin{array}{l}\text { Laparoscopy with deep neuromuscular blockade and low } \\
\text { pressure }(n=49) \text { or standard pressure }(n=50) \text {. } \\
\text { Baseline: acetaminophen, etodolac. }\end{array}$ & $\begin{array}{l}\text { Abdominal pain similar, incidence of } \\
\text { shoulder tip pain lower in low-pressure } \\
\text { group. }\end{array}$ & NS \\
\hline $\begin{array}{l}\text { Herrmann and De } \\
\text { Wilde }^{66}\end{array}$ & $\begin{array}{l}\text { Laparoscopy using humidified and heated } \mathrm{CO}_{2}(\mathrm{n}=48) \text { or controls } \\
(\mathrm{n}=49) \text {. } \\
\text { Baseline analgesia: metamizole. }\end{array}$ & NS & Higher in control group \\
\hline Radosa et al ${ }^{67}$ & $\begin{array}{l}\text { Active } \mathrm{CO}_{2} \text { elimination }(\mathrm{n}=98), \mathrm{CO}_{2} \text { elimination plus trocar site } \\
\text { infiltration }(n=95) \text {, or control }(n=96) . \\
\text { Baseline analgesia: metamizole. }\end{array}$ & $\begin{array}{l}\text { Higher in the control group by } 0.5-1.7 \\
\text { NRS points at } 3,24 \text {, and } 48 \text { hours. }\end{array}$ & $\begin{array}{l}\text { Higher piritramide requirement in the } \\
\text { control group during the first } 24 \text { hours. }\end{array}$ \\
\hline Roy et $a l^{68}$ & $\begin{array}{l}\text { TLH }(n=30) \text { vs LAVH ( } n=30) \text {, vs non-descent vaginal hysterectomy } \\
(n=30) \text {. } \\
\text { Baseline analgesia: not reported. }\end{array}$ & NS & \\
\hline Fagotti et al ${ }^{69}$ & $\begin{array}{l}\text { Thunderbeat electrosurgery or conventional cautery }(n=25 \\
\text { patient per group). } \\
\text { Baseline analgesia: acetaminophen. }\end{array}$ & $\begin{array}{l}\text { Lower pain scores by } 1-1.5 \text { NRS points } \\
\mathrm{mm} \text { in the Thunderbeat group. }\end{array}$ & $\begin{array}{l}\text { More patients requiring rescue analgesics } \\
\text { in the conventional group. }\end{array}$ \\
\hline Rothmund et $\mathrm{al}^{70}$ & $\begin{array}{l}\text { Standard bipolar }(n=80) \text { vs EnSeal cautery }(n=80) \text {. } \\
\text { Baseline analgesia: not reported. }\end{array}$ & NS & \\
\hline
\end{tabular}

Empty fields denote variable not reported.

$\mathrm{CO}_{2}$, carbon dioxide; LAVH, laparoscopic-assisted vaginal hysterectomy; LH, laparoscopic hysterectomy; MLH, minilaparoscopic hysterectomy; NRS, Numerical Rating Scale; NS, no significant difference between groups; NSAID, non-steroidal anti-inflammatory drug; PCA, patient-controlled analgesia; POD, postoperative day; TAP, transversus abdominis plane; TLH, total laparoscopic hysterectomy.

receiving 0.75 and $1 \mu \mathrm{g} / \mathrm{kg}$ body weight, respectively. A study compared intravenous infusions of dexmedetomidine/ketorolac $(n=25)$ with remifentanil/ketorolac $(n=25)$ administered from the end of surgery to the arrival in the PACU. ${ }^{25}$ The early VAS scores were not significantly different between the two groups until $30 \mathrm{~min}$ after PACU arrival, when the study ended. In conclusion, there are insufficient data to form a recommendation for dexmedetomidine. When Choi and colleagues ${ }^{26}$ compared different brief analgesic infusions at the end of surgery, they found no difference in pain scores or analgesic side effects between remifentanil, fentanyl, and dexmedetomidine.

\section{Opioids}

Several studies compared different variations of opioids in their ability to control pain. These studies compared intravenous PCA morphine versus oxycodone, ${ }^{27}$ fentanyl versus oxycodone, ${ }^{28-30}$ and brief infusions of remifentanil ${ }^{31}$ or sufentanil ${ }^{32}$ before extubation. There is insufficient evidence to specifically recommend one opioid over another. Observational studies suggest that most patients after laparoscopic hysterectomy require opioids as rescue drugs, for a median of 4 days. $^{6}$

\section{Regional anesthesia/analgesia Epidural analgesia}

The pre-emptive analgesic effects of epidural analgesia were compared with epidural analgesia started after skin closure in patients with cervical cancer scheduled for laparoscopic hysterectomy. Continuous epidural analgesia using a patient-controlled epidural analgesia (PCEA, lidocaine and morphine) was continued for 72 hours. In the pre-emptive epidural analgesia group, pain scores and rescue PCEA opioid consumption were lower, and side effects nausea, vomiting, and pruritus were reduced, as compared with epidural analgesia initiated after the end of surgery. ${ }^{33}$ During surgery, after the initial top-up in the pre-emptive group, blood pressure was slightly lower than baseline, while in the group that had not received an epidural bolus blood pressure was higher than baseline. The overall recovery effect of this 72-hour regimen compared with other simple multimodal opioid-sparing techniques was not determined. Patients in the pre-emptive epidural group had median pain scores of less than 4 after surgery and less than 3 at 3 hours postoperatively, whereas the control group reached similar pain control only between 12 and 24 hours after surgery.

\section{Transversus abdominis plane block}

The efficacy of ultrasound-guided transversus abdominis plane (TAP) block was assessed in several studies. De Oliveira and coworkers ${ }^{34}$ compared two concentrations of ropivacaine $(5 \mathrm{mg} /$ $\mathrm{mL}$ or $2.5 \mathrm{mg} / \mathrm{mL}$ ) with saline for TAP block and found lower NRS pain scores in the ropivacaine groups by $2-3$ points in the PACU, and at 24 hours, and in addition lower opioid consumption in the $5 \mathrm{mg} / \mathrm{mL}$ ropivacaine group as compared with saline (7.5 vs $15 \mathrm{mg}$ morphine equivalent). Importantly, this study used standard multimodal analgesia (opioid, acetaminophen, ibuprofen) in all patients.

When comparing TAP block with no block, Kane and colleagues ${ }^{35}$ found no differences in pain scores or opioid requirements. In this study, intraoperative and postoperative titration of analgesics was at the discretion of the treating doctors, and all patients received NSAID. Calle and colleagues ${ }^{36}$ compared TAP block with placebo in the setting of ambulatory laparoscopic hysterectomy and found a lower VAS score at discharge in the TAP group (by less than 1 point on the NRS), which was statistically but not clinically relevant, while no difference was observed at 12 or 24 hours when patients received a standard baseline analgesic regimen including ibuprofen and acetaminophen. Of note, the TAP block in this study was performed using the loss-of-resistance technique without ultrasound, under laparoscopic vision. 
In another study, when compared with trocar site infiltration in the setting of multimodal analgesia, single-shot ultrasound-guided TAP block decreased neither pain scores to a clinically significant extent (mean difference less than 1 point on the NRS), nor intraoperative or postoperative opioid use or antiemetic prescription. ${ }^{37}$ Torup and colleagues ${ }^{38}$ investigated the use of TAP block when added to multimodal analgesia (two non-opioids, morphine PCA) and found no additional benefit concerning pain scores when compared with placebo blocks, no reduction in opioid use, and no difference in adverse events. Hutchins and colleagues ${ }^{39}$ investigated the use of liposomal bupivacaine compared with plain bupivacaine for subcostal TAP block in patients undergoing robotic-assisted laparoscopic hysterectomy. There was no clinically significant difference in median maximal pain scores in the immediate postoperative period, but the liposomal bupivacaine group had decreased maximum pain scores, decreased opioid use, and a lower incidence of PONV for the first 24 hours. ${ }^{39}$ Ghisi and coworkers ${ }^{40}$ compared ultrasound-guided TAP block with systemic opioid analgesia and found no advantage in terms of VAS score, opioid consumption, postoperative mobilization, or adverse events. Finally, Guardabassi and colleagues ${ }^{41}$ found no advantage in terms of opioid consumption during the first 24 hours, and no difference in pain scores, when TAP blocks were added to a systemic analgesia regimen including opioid PCA.

\section{Intraperitoneal local anesthetics}

Two studies investigating different regimens of local anesthetics used for intraperitoneal instillation of local anesthetics demonstrated no clinically relevant benefit. Arden and coworkers ${ }^{42}$ compared the instillation of local anesthetic in the pelvic region, but not the trocar insertion sites, with saline placebo and found no difference in VAS scores or adverse events. Andrews and colleagues $^{43}$ investigated continuous intraperitoneal infusion of $5 \mathrm{mg} / \mathrm{mL}$ levobupivacaine at $2 \mathrm{~mL} /$ hour for 48 hours as compared with saline placebo, and found higher postoperative analgesic requests in the levobupivacaine group while attaining comparable pain scores.

\section{Port site infiltration}

As indicated above, the efficacy of bupivacaine port site infiltration and ketorolac administration was compared in a four-arm study, where the lowest pain scores during the first 6 hours following surgery were found in the group which received both port site infiltration and ketorolac. ${ }^{16}$ Also, in the setting of multimodal analgesia, patients receiving trocar site infiltration reported the same (low) pain scores as patients receiving singleshot ultrasound-guided TAP blocks. ${ }^{37}$ Barron and colleagues ${ }^{44}$ showed that port site infiltration with liposomal bupivacaine, when compared with plain bupivacaine, showed lower pain scores on postoperative days 2 and 3, with no difference in opioid consumption, psychometrics, or analgesic side effects.

In summary, based on one well-designed study, epidural analgesia started before skin incision provided adequate analgesia postoperatively. TAP blocks were less effective, with four out of seven studies negative, two studies positive on the margin of clinical significance, and one positive study. Liposomal bupivacaine for TAP block, in one study, seemed to have a prolonged effect, and lowered worst pain scores during first 24 hours, as well as opioid consumption. Intraperitoneal local anesthetic infusion showed no analgesic benefits in the setting of laparoscopic hysterectomy. There is no procedure-specific evidence for port site infiltration.

\section{Anesthetic interventions}

In a study comparing isoflurane and propofol for maintenance of general anesthesia, pain scores and postoperative analgesic requirements were comparable in the two groups, ${ }^{45}$ and in a similar study no difference was found when anesthesia was maintained with sevoflurane versus propofol. ${ }^{46}$ Finally, a single antiemetic dose of propofol at the end of surgery did not influence pain scores or opioid requirements. ${ }^{47}$

\section{Surgical interventions}

Surgical techniques are chosen based on anatomic and patient-centered factors, with postoperative pain scores playing a lesser role. Laparoscopic-assisted vaginal hysterectomy (LAVH) was shown in one study to lead to less pain compared with minilaparotomy, ${ }^{48}$ while the difference in a second study was minimal. ${ }^{49}$ Eggemann and colleagues ${ }^{50}$ found only minor differences in pain (less than 1 point on the NRS) when vaginal hysterectomy was compared with LAVH. Studies comparing single-port surgery versus minilaparotomy were mixed, with one study reporting less pain in the single-port group, ${ }^{51}$ while others finding no difference. ${ }^{5253}$ Chung and colleagues ${ }^{54}$ reported more rescue analgesics in the single-port group when it was compared with a standard three-port surgery while other studies found no difference in pain scores or analgesics. ${ }^{55} 56$ Compared with single-port hysterectomy, minilaparoscopy led to lower pain scores in one study. ${ }^{57}$ No difference in pain scores and analgesics was found when robotic and conventional laparoscopic hysterectomy were compared. ${ }^{58} 59$ Laparoscopic combined hysterectomy was not different from conventional hysterectomy. ${ }^{60}$ Minilaparoscopy compared with conventional (larger-bore) hysterectomy led to no ${ }^{61}$ or only minimal ${ }^{62}$ decreases in pain scores.

\section{Pneumoperitoneum, drains, and cautery}

A study compared closed suction (Jackson-Pratt) drains with no drains. ${ }^{63}$ Shoulder tip, abdominal, and back pain were evaluated by measuring VAS scores at 3 hours, 24 hours, and 48 hours after surgery. Closed suction drains reduced shoulder tip pain at 24 hours. At 48 hours, fewer women who received drains experienced abdominal pain. There were no statistically significant differences in VAS scores for back pain at any time point. The oral analgesics demand was higher in patients with no drain.

Pain after pneumoperitoneum was investigated by several studies. Bogani and colleagues ${ }^{64}$ compared low-pressure (8 $\mathrm{mm} \mathrm{Hg}$ ) versus standard-pressure $(12 \mathrm{~mm} \mathrm{Hg})$ pneumoperitoneum and found that while abdominal pain was similar between groups, the incidence of shoulder tip pain in the early postoperative period was 36\% in the standard and 5\% in the low-pressure group. Madsen and colleagues ${ }^{65}$ demonstrated less shoulder tip pain with lower inflation pressure. When carbon dioxide $\left(\mathrm{CO}_{2}\right)$ was humidified and heated, postoperative shoulder tip pain scores, but not abdominal pain scores, were lower than when using control gases. ${ }^{66}$ Lastly, elimination of $\mathrm{CO}_{2}$ with an open umbilical trocar decreased postoperative pain scores, but additional trocar site infiltration did not decrease pain scores or opioid consumption further. ${ }^{67}$ In one study, there were no differences in postoperative pain and adverse events between total laparoscopic hysterectomy, LAVH, and non-descent vaginal hysterectomy. ${ }^{68}$

Fagotti and colleagues ${ }^{69}$ compared postoperative pain and operative time when using conventional laparoscopic cautery, or a combined multifunctional instrument (Thunderbeat, Olympus, Tokyo, Japan), and found a shorter surgery time, but only small differences in postoperative pain. Similarly, no difference in 
postoperative pain scores was found when an EnSeal (Ethicon, Norderstedt, Germany) was compared with conventional bipolar coagulation. $^{70}$

In summary, limited data suggest that small-port laparoscopy may decrease early and short-term postoperative pain, and low inflation pressure or humidified and heated $\mathrm{CO}_{2}$ may decrease shoulder tip pain, but not abdominal pain.

\section{DISCUSSION}

This systematic review examined the effects of analgesic, anesthetic, and surgical techniques on postoperative pain after laparoscopic hysterectomy.

Based on the available studies, non-opioids (acetaminophen and NSAIDs) have opioid-sparing effects, but in the procedure-specific studies they did not reduce opioid side effects. The number of patients included was too small to draw valid conclusions concerning their safety profile in the settings of laparoscopic hysterectomy. The combination of port site infiltration and NSAID resulted in a better analgesia than either technique alone in one small study ${ }^{16}$ and showed equivalence to TAP blocks in another. ${ }^{37}$ Based on the origin and the type and duration of pain after laparoscopic hysterectomy, as well as the available evidence from procedure-specific RCTs, it is recommended to administer acetaminophen and NSAID combination to all patients unless there are contraindications. The studies supporting the opioidsparing effect were of generally good quality, but study size was small in all but one study. ${ }^{15}$ This approach is supported by a recent network meta-analysis on patients after major surgery. ${ }^{71}$

Dexamethasone was shown to reduce opioids in two studies which were of higher quality, ${ }^{19}{ }^{20}$ while one study with lower quality $^{21}$ found no effect. Effective doses seemed to be in the range of $8-10 \mathrm{mg}$. Even though the timing of dexamethasone administration has not been specifically investigated in the setting of laparoscopic hysterectomy, evidence from visceral abdominal surgery suggests that early administration after anesthetic induction may offer the best antiemetic, ${ }^{72}$ anti-inflammatory, and analgesic effects. ${ }^{73}$

Pregabalin was found in two well-designed trials 2223 to be opioid-sparing, but specific side effects (blurred vision, dizziness) were also increased. It should be noted, however, that the doses used were rather high (up to $600 \mathrm{mg}$ ). More evidence is needed to determine the potential beneficial effects of alpha-2-agonists, despite promising results in one trial. ${ }^{24}$

Evidence on epidural analgesia was limited, while evidence on TAP blocks was inconsistent, and that for intraperitoneal local anesthetics was negative. Epidural analgesia provides excellent pain relief for patients undergoing laparoscopic hysterectomy, but given the fact that surgery is now often performed on an ambulatory basis, and the adequacy of less invasive modalities in managing pain in most patients, it is performed very rarely ${ }^{74}$ and should be considered a reserve intervention. Several studies evaluated the use of TAP blocks for laparoscopic hysterectomy; however, the results are conflicting. When added to non-opioids and opioids, or compared with a simpler procedure such as port site infiltration, ${ }^{37}$ TAP block failed to consistently yield clinically significant benefits. Quality of studies did not seem to differentiate between positive and negative outcomes. One potential reason for the failure of TAP blocks in some trials may be the localization of port sites; depending on the size of the uterus and pathology, these may be placed at the level of and below the umbilicus, or above the umbilicus, and as mentioned others may use midline single-port techniques. ${ }^{35}$ So lateral TAP blocks may not be optimally suited for all types of laparoscopic
Box 1 Overall recommendations for pain management in patients undergoing laparoscopic hysterectomy

Perioperative interventions in time to secure analgesia in immediate postoperative period.

- Acetaminophen.

- Non-selective NSAID or COX-2 selective inhibitor.

- Single dose of dexamethasone, intravenously.

Postoperative period.

- Acetaminophen (paracetamol) and NSAID or COX-2 selective inhibitor.

- Rescue opioid.

COX, cyclo-oxygenase; NSAID, non-steroidal anti-inflammatory drug.

hysterectomy, and subcostal blocks may be more appropriate for patients with more cranial port insertion points. There was also heterogeneity in block performance; some blocks were performed before surgery, while some others were performed after skin closure, and Hutchins and colleagues ${ }^{39}$ used liposomal bupivacaine. There were not enough studies to differentiate further whether any particular variant of TAP block might be more effective than others, such that a universal recommendation cannot be given at this point.

Intraperitoneal instillation of local anesthetic provided no clinically significant benefit in two studies with good quality and adequate sample size. ${ }^{42} 43$ At the present time, there is no procedure-specific evidence of benefit of port site infiltration for laparoscopic hysterectomy, although we note that significant benefit has been demonstrated for this intervention for laparoscopic cholecystectomy. ${ }^{75}$

Analgesics adjuncts which have been suggested as potential options include ketamine and intravenous lidocaine infusion. These approaches are not recommended for laparoscopic hysterectomy because of inadequate procedure-specific evidence. In addition, the benefit of adding these drugs to the recommended pain management regimen (box 1) has not been evaluated.

General anesthesia is the standard of care for laparoscopic hysterectomy, and from an acute pain standpoint the choice of maintenance anesthetics has no detectable effect on postoperative pain, such that the main determinants for choosing the maintenance anesthetic agents are patient-related, such as cardiovascular comorbidity and the risk of PONV.

With respect to the surgical technique, limited data suggest that minilaparoscopy may lead to lower postoperative pain scores. Measures to lower peritoneal insufflation pressure or humidify or heat insufflated gas may help to reduce the incidence of shoulder tip, but not abdominal, pain.

All these considerations need to be taken into account with the timeline of patients moving through the perioperative period. Between 2005 and 2013, the incidence of laparoscopic hysterectomy increased from less than $20 \%$ to account for more than two-thirds of all hysterectomies in the USA. ${ }^{76}$ When divided by subgroup, the largest increase was observed in robotic-assisted laparoscopic hysterectomy, while the number of single-port laparoscopic surgeries was negligible. ${ }^{76}$ The overall initial postoperative pain can be severe, ${ }^{7}$ but generally subsides to NRS scores $<4$ within 1 day. ${ }^{77}$ Pain generated from laparoscopic hysterectomy includes incisional pain, which can be severe initially, but subsides within the first half day; visceral pain, which takes longer (up to a day) to resolve; and shoulder pain, which is milder, typically 
appears within 24 hours and can last for several days. ${ }^{7}$ Typical length for use of opioid rescue drugs is approximately 4 days in a recent US study. ${ }^{6}$ Laparoscopic hysterectomies are increasingly performed as ambulatory procedures, ${ }^{78}$ emphasizing the need for standardized pain management on discharge. In this light, continuous or invasive methods such as epidural analgesia and intravenous opioid PCA may not be suited for prospective ambulatory patients and should be regarded reserve interventions. Lastly, as mentioned above, robotic surgery is increasingly used to perform laparoscopic hysterectomy, ${ }^{79}$ but the two studies investigating pain scores found no advantage in terms of pain as such over conventional laparoscopic hysterectomy. ${ }^{58} 59$

Our study confirms and expands on the results of a prior systematic review focusing on non-opioid analgesics. ${ }^{8}$ Similar to this study, we found opioid-sparing effects of non-opioids such as acetaminophen, NSAIDs, and dexamethasone, and ambivalent evidence for regional techniques. We respectfully disagree with the authors of this systematic review ${ }^{8}$ on the risk-benefit analysis of anticonvulsants, the opioid-sparing effects of which need to be weighed against increased risk of sedative side effects.

\section{Strengths}

The strength of our systematic review comes from the interpretation of the individual pieces of literature according to a predetermined methodology of the PROSPECT Working Group, with an interdisciplinary Delphi process to achieve consensus. ${ }^{90}$ This approach goes beyond a simple summation of results and seeks to interpret on the basis of background/baseline analgesic technique, side effects, and clinical context.

\section{Limitations}

The limitations of this study are related to the limitations of the included studies. Most of the included studies assessed "unimodal" analgesic therapies rather than broader and more comprehensive analgesic techniques. Ideally, control groups should receive an optimized analgesic regimen, and the added procedure-specific benefit of specific interventions should then be tested against this backdrop. ${ }^{81}$ Also, there was significant heterogeneity in study designs with respect to the analgesic regimen. The minimal clinically important difference (MCID) in pain score has been debated, and while some have suggested a cut-off of $20 \mathrm{~mm}$ on a $100 \mathrm{~mm}$ VAS, or $30 \%$ on the NRS, ${ }^{82}$ some have argued for $10 \mathrm{~mm}^{14}$ and others have advocated individualizing MCID to baseline pain. ${ }^{83}$ We chose $10 \mathrm{~mm}$ as our defined MCID in accordance with the recent study by Myles and colleagues, ${ }^{14}$ who postulated $10 \mathrm{~mm}$ to be the MCID, and an absolute score of $33 \mathrm{~mm}$ on a $100 \mathrm{~mm}$ VAS to signify "acceptable pain control." Although most studies report pain at rest, studies reporting pain on activity would be more suited to judge recovery from surgery with respect to mobilization.

\section{Future outlook}

The primary outcome measures for systematic reviews by the PROSPECT Working Group are pain and opioid consumption. Although these parameters have been used in clinical research for the past decades, future studies and systematic reviews need to link these measures of pain with more far-reaching outcome parameters, such as mobilization, length of hospital stay, the incidence of complications, and the occurrence of chronic pain or chronic opioid consumption. Summarizing these considerations, we often do not use the most effective method to treat pain (eg, combined femoral and sciatic nerve blocks for knee arthroplasty), ${ }^{84}$ but the one we consider most appropriate after weighing risks and benefits. ${ }^{85}$ The present recommendations take this into account by suggesting a framework of basic analgesic interventions, supplemented by rescue medications, that can serve as a gold standard which can readily be implemented and against the backdrop of which future interventions need to be tested. ${ }^{10}$ Also, as individualized treatment plans seek to take into account patient-specific factors such as chronic pain, ${ }^{6}$ or pre-existing opioid therapy, ${ }^{86}$ our recommendations can serve as the common basis from which to tailor and adapt analgesic plans.

\section{CONCLUSIONS}

In summary, although considered less painful than open abdominal hysterectomy, laparoscopic hysterectomy requires standardized postoperative pain management, particularly in the early postoperative period. The PROSPECT recommendations provide clinicians with supporting arguments for and against the use of analgesic interventions for laparoscopic hysterectomy (table 3). A balance of the analgesic efficacy and potential risks of the analgesic intervention determines these recommendations. Perioperative pain treatment for laparoscopic hysterectomy should include, unless contraindicated, acetaminophen and an NSAID continued into the postoperative period primarily for their ability to reduce opioid use, dexamethasone for its ability to decrease analgesic use and act as an antiemetic, and opioids as rescue postoperatively.

Collaborators H Beloeil, A Hill, H Kehlet, P Lavand'homme, E Pogatzki-Zahn, N Rawal, J Raeder, S Schug, M van de Veldex

Contributors JT, PL, and M-PB conducted the literature search and analyzed the retrieved articles with FB. JT, PL, FB, and GPJ wrote the manuscript, which was reviewed and edited by all the other authors, who have also participated in the PROSPECT Working Group meetings using the Delphi method, and in defining the methodology of the PROSPECT group.

Funding PROSPECT is supported by an unrestricted grant from the European Society of Regional Anaesthesia and Pain Therapy (ESRA). In the past, PROSPECT had received unrestricted grants from Pfizer (New York, New York, USA) and Grunenthal (Aachen, Germany).

Competing interests GPJ has received honoraria from Baxter, Mallinckrodt, Pacira, and Merck Pharmaceuticals. FB has received honoraria from Pfizer, The Medicines Company, Abbott France, and Nordic Pharma France. Henrik Kehlet has received honoraria from Pfizer and Grunenthal. The Anesthesiology Unit of the University of Western Australia, but not Stephan Schug privately, has received research and travel funding and speaking and consulting honoraria from bioCSL, Eli Lilly, Indivior, iX Biopharma, and Pfizer within the last 2 years. Narinder Rawal has received honoraria from Baxter and Sintetica.

Patient consent for publication Not required.

Provenance and peer review Not commissioned; externally peer reviewed.

Data sharing statement All data will be available on the website: www. postoppain.org.

Open access This is an Open Access article distributed in accordance with the Creative Commons Attribution Non Commercial (CC BY-NC 4.0) license, which permits others to distribute, remix, adapt, build upon this work non-commercially, and license their derivative works on different terms, provided the original work is properly cited, an indication of whether changes were made, and the use is noncommercial. See: http://creativecommons.org/licenses/by-nc/4.0/.

\section{REFERENCES}

1 Gendy R, Walsh CA, Walsh SR, et al. Vaginal hysterectomy versus total laparoscopic hysterectomy for benign disease: a metaanalysis of randomized controlled trials. Am J Obstet Gynecol 2011;204:388.e1-8.

2 Walsh CA, Walsh SR, Tang TY, et al. Total abdominal hysterectomy versus total laparoscopic hysterectomy for benign disease: a meta-analysis. Eur J Obstet Gynecol Reprod Biol 2009;144:3-7

3 Bijen CBM, Vermeulen KM, Mourits MJE, et al. Costs and effects of abdominal versus laparoscopic hysterectomy: systematic review of controlled trials. PLoS One 2009;4:e7340 
4 Mourits MJE, Bijen CB, Arts HJ, et al. Safety of laparoscopy versus laparotomy in earlystage endometrial cancer: a randomised trial. Lancet Oncol 2010;11:763-71.

5 YX Y, Zhang W, Zhou Q, et al. Laparoscopic-assisted vaginal hysterectomy vs abdominal hysterectomy for benign disease: a meta-analysis of randomized controlled trials. Eur J Obstet Gynecol Reprod Biol 2011;159:1-18.

6 As-Sanie S, Till SR, Mowers EL, et al. Opioid prescribing patterns, patient use, and postoperative pain after hysterectomy for benign indications. Obstet Gynecol 2017:130:1261-8.

7 Choi JB, Kang K, Song MK, et al. Pain characteristics after total laparoscopic hysterectomy. Int J Med Sci 2016;13:562-8.

8 Blanton E, Lamvu G, Patanwala I, et al. Non-opioid pain management in benign minimally invasive hysterectomy: A systematic review. Am J Obstet Gynecol 2017:216:557-67.

9 Joshi GP, Schug SA, Kehlet H. Procedure-specific pain management and outcome strategies. Best Pract Res Clin Anaesthesiol 2014;28:191-201.

10 Joshi GP, Kehlet H, Beloeil H, et al. Guidelines for perioperative pain management: need for re-evaluation. Br J Anaesth 2017:119:720-2.

11 Moher D, Liberati A, Tetzlaff J, et al. Preferred reporting items for systematic reviews and meta-analyses: the PRISMA statement. J Clin Epidemiol 2009;62:1006-12.

12 Neugebauer EAM, Wilkinson RC, Kehlet $H$, et al. Prospect: a practical method for Formulating evidence-based expert recommendations for the management of postoperative pain. Surg Endosc 2007;21:1047-53.

13 Jadad AR, Moore RA, Carroll D, et al. Assessing the quality of reports of randomized clinical trials: is blinding necessary? Control Clin Trials 1996;17:1-12.

14 Myles PS, Myles DB, Galagher W, et al. Measuring acute postoperative pain using the visual analog scale: the minimal clinically important difference and patient acceptable symptom state. Br J Anaesth 2017;118:424-9.

15 Jokela R, Ahonen J, Seitsonen $\mathrm{E}$, et al. The influence of ondansetron on the analgesic effect of acetaminophen after laparoscopic hysterectomy. Clin Pharmacol Ther 2010:87:672-8

$16 \mathrm{Kim} \mathrm{JH}$, Lee YS, Shin HW, et al. Effect of administration of ketorolac and local anaesthetic infiltration for pain relief after laparoscopic-assisted vaginal hysterectomy. Int Med Res 2005;33:372-8.

$17 \mathrm{Kim} \mathrm{J-J,} \mathrm{Ha} \mathrm{M-H,} \mathrm{Jung} \mathrm{S-H,} \mathrm{et} \mathrm{al.} \mathrm{The} \mathrm{efficiency} \mathrm{of} \mathrm{IV} \mathrm{PCA} \mathrm{with} \mathrm{remifentanil} \mathrm{and}$ ketorolac after laparoscopic-assisted vaginal hysterectomy. Korean I Anesthesiol 2011;61:42-9

18 Moon JY, Choi SS, Lee SY, et al. The effect of nefopam on postoperative fentanyl consumption: a randomized, double-blind study. Korean J Pain 2016;29:110-8.

19 Jokela RM, Ahonen JV, Tallgren MK, et al. The effective analgesic dose of dexamethasone after laparoscopic hysterectomy. Anesth Analg 2009;109:607-15.

20 Thangaswamy CR, Rewari V, Trikha A, et al. Dexamethasone before total laparoscopic hysterectomy: a randomized controlled dose-response study. J Anesth 2010;24:24-30.

$21 \mathrm{Nam} \mathrm{M}$, Yoon H. Effect of Ondansetron combined with Dexamethasone on Postoperative Nausea \& Vomiting and Pain of Patients with Laparoscopic Hysterectomy. J Korean Acad Nurs 2009:39:44-52.

22 Jokela R, Ahonen J, Tallgren M, et al. A randomized controlled trial of perioperative administration of pregabalin for pain after laparoscopic hysterectomy. Pain 2008;134:106-12.

23 Asgari Z, Rouholamin S, Nataj M, et al. Dose ranging effects of pregabalin on pain in patients undergoing laparoscopic hysterectomy: a randomized, double blinded, placebo controlled, clinical trial. J Clin Anesth 2017;38:13-17.

24 Kim Y, Kim Y, Seo K, et al. Optimal dose of prophylactic dexmedetomidine for preventing postoperative shivering. Int J Med Sci 2013;10:1327-32.

25 Jung HS, Joo JD, Jeon YS, et al. Comparison of an intraoperative infusion of dexmedetomidine or remifentanil on perioperative haemodynamics, hypnosis and sedation, and postoperative pain control. J Int Med Res 2011:39:1890-9.

26 Choi JW, Joo J-D, Kim D-W, et al. Comparison of an intraoperative infusion of dexmedetomidine, fentanyl, and remifentanil on perioperative hemodynamics, sedation quality, and postoperative pain control. J Korean Med Sci 2016:31:1485-90.

27 Lenz H, Sandvik L, Qvigstad E, et al. A comparison of intravenous oxycodone and intravenous morphine in patient-controlled postoperative analgesia after laparoscopic hysterectomy. Anesth Analg 2009;109:1279-83.

$28 \mathrm{Kim} \mathrm{N-S}$, Kang KS, Yoo SH, et al. A comparison of oxycodone and fentanyl in intravenous patient-controlled analgesia after laparoscopic hysterectomy. Korean J Anesthesiol 2015;68:261-6.

29 Kim NS, Lee JS, Park SY, et al. Oxycodone versus fentanyl for intravenous patientcontrolled analgesia after laparoscopic supracervical hysterectomy: a prospective randomized, double-blind study. Medicine 2017:96:e6286.

30 Park J-H, Lee C, Shin Y, et al. Comparison of oxycodone and fentanyl for postoperative patient-controlled analgesia after laparoscopic gynecological surgery. Korean $J$ Anesthesiol 2015;68:153-8

31 Lee JJ, Hwang SM, Lee JS, et al. Continuous infusion of two doses of remifentanil immediately after laparoscopic-assisted vaginal hysterectomy. Korean J Anesthesiol 2010;58:537-41.

32 Lee JY, Lim BG, Park HY, et al. Sufentanil infusion before extubation suppresses coughing on emergence without delaying extubation time and reduces postoperative analgesic requirement without increasing nausea and vomiting after desflurane anesthesia. Korean J Anesthesiol 2012;62:512-7.

33 Hong J-Y, Lim KT. Effect of preemptive epidural analgesia on cytokine response and postoperative pain in laparoscopic radical hysterectomy for cervical cancer. Reg Anesth Pain Med 2008;33:44-51

34 De Oliveira GS, Milad MP, Fitzgerald P, et al. Transversus abdominis plane infiltration and quality of recovery after laparoscopic hysterectomy. Obstet Gynecol 2011:118:1230-7.

35 Kane SM, Garcia-Tomas V, Alejandro-Rodriguez M, et al. Randomized trial of transversus abdominis plane block at total laparoscopic hysterectomy: effect of regional analgesia on quality of recovery. Am J Obstet Gynecol 2012;207:419. e1-5.

36 Calle GA, López CC, Sánchez E, et al. Transversus abdominis plane block after ambulatory total laparoscopic hysterectomy: randomized controlled trial. Acta Obstet Gynecol Scand 2014:93:345-50.

37 El Hachem L, Small E, Chung P, et al. Randomized controlled double-blind trial of transversus abdominis plane block versus trocar site infiltration in gynecologic laparoscopy. Am J Obstet Gynecol 2015;212:182.e1-9.

38 Torup H, Bøgeskov M, Hansen EG, et al. Transversus abdominis plane (TAP) block after robot-assisted laparoscopic hysterectomy: a randomised clinical trial. Acta Anaesthesiol Scand 2015;59:928-35.

39 Hutchins J, Delaney D, Vogel RI, et al. Ultrasound guided subcostal transversus abdominis plane (TAP) infiltration with liposomal bupivacaine for patients undergoing robotic assisted hysterectomy: a prospective randomized controlled study. Gynecol Oncol 2015;138:609-13.

40 Ghisi D, Fanelli A, Vianello F, et al. Transversus abdominis plane block for postoperative analgesia in patients undergoing total laparoscopic hysterectomy: a randomized, controlled, observer-blinded trial. Anesth Analg 2016;123:488-92.

41 Guardabassi DS, Lupi S, Agejas R, et al. Efficacy of ultrasound-guided transversus abdominis plane block in laparoscopic hysterectomy. clinical trial. Rev Esp Anestesiol Reanim 2017;64:257-61.

42 Arden D, Seifert E, Donnellan N, et al. Intraperitoneal instillation of bupivacaine for reduction of postoperative pain after laparoscopic hysterectomy: a double-blind randomized controlled trial. J Minim Invasive Gynecol 2013;20:620-6.

43 Andrews V, Wright JT, Zakaria F, et al. Continuous infusion of local anaesthetic following laparoscopic hysterectomy-a randomised controlled trial. BJOG: Int J Obstet Gy 2014;121:754-60.

44 Barron KI, Lamvu GM, Schmidt RC, et al. Wound infiltration with extended-release versus short-acting bupivacaine before laparoscopic hysterectomy: a randomized controlled trial. J Minim Invasive Gynecol 2017;24:286-92.

45 Nelskyla K, Eriksson H, Soikkeli A, et al. Recovery and outcome after propofol and isoflurane anesthesia in patients undergoing laparoscopic hysterectomy. Acta Anaesthesiol Scand 1997:41:360-3.

46 Pokkinen SM, Yli-Hankala A, Kalliomaki ML. The effects of propofol vs. sevoflurane on post-operative pain and need of opioid. Acta Anaesthesiol Scand 2014;58:980-5.

47 Kim E-G, Park HJ, Kang H, et al. Antiemetic effect of propofol administered at the end of surgery in laparoscopic assisted vaginal hysterectomy. Korean J Anesthesiol 2014;66:210-5.

48 Muzii L, Basile S, Zupi E, et al. Laparoscopic-assisted vaginal hysterectomy versus minilaparotomy hysterectomy: a prospective, randomized, multicenter study. J Minim Invasive Gynecol 2007;14:610-5.

49 Song T, Kim MK, Kim M-L, et al. A randomized comparison of laparoendoscopic singlesite hysterectomies: total laparoscopic hysterectomy versus laparoscopically assisted vaginal hysterectomy. J Laparoendosc Adv Surg Tech A 2015;25:541-7.

50 Eggemann $\mathrm{H}$, Ignatov $\mathrm{A}$, Frauchiger-Heuer $\mathrm{H}$, et al. Laparoscopic-assisted vaginal hysterectomy versus vaginal hysterectomy for benign uterine diseases: a prospective, randomized, multicenter, double-blind trial (lava). Arch Gynecol Obstet 2018;297:479-85.

51 Chen YJ, Wang PH, Ocampo EJ, et al. Single-port compared with conventional laparoscopic-assisted vaginal hysterectomy: a randomized controlled trial. Obstet Gynecol 2011:117:906-12.

52 Li M, Han Y, Feng YC. Single-port laparoscopic hysterectomy versus conventional laparoscopic hysterectomy: a prospective randomized trial. J Int Med Res 2012:40:701-8

53 Jung YW, Lee M, Yim GW, et al. A randomized prospective study of single-port and four-port approaches for hysterectomy in terms of postoperative pain. Surg Endosc 2011;25:2462-9

54 Chung J-H, Baek JM, Chung K, et al. A comparison of postoperative pain after transumbilical single-port access and conventional three-port total laparoscopic hysterectomy: a randomized controlled trial. Acta Obstet Gynecol Scand 2015;94:1290-6.

$55 \mathrm{Kim}$ TJ, Shin SJ, Kim TH, et al. Multi-institution, prospective, randomized trial to compare the success rates of single-port versus multiport laparoscopic hysterectomy for the treatment of uterine myoma or adenomyosis. J Minim Invasive Gynecol 2015:22:785-91.

56 Song T, Cho J, Kim T-J, et al. Cosmetic outcomes of laparoendoscopic single-site hysterectomy compared with multi-port surgery: randomized controlled trial. J Minim Invasive Gynecol 2013;20:460-7. 
57 Fanfani F, Fagotti A, Gagliardi ML, et al. Minilaparoscopic Versus Single-Port Total Hysterectomy: A Randomized Trial. J Minim Invasive Gynecol 2013;20:192-7.

58 Paraiso MFR, Ridgeway B, Park AJ, et al. A randomized trial comparing conventional and robotically assisted total laparoscopic hysterectomy. American J Obstet Gynecol 2013;208:368.e1-7.

59 Sarlos D, Kots L, Stevanovic N, et al. Robotic compared with conventional laparoscopic hysterectomy: a randomized controlled trial. Obstet Gynecol 2012:120:604-11.

60 Tchartchian G, Heldmann P, Bojahr B, et al. The laparoscopic-assisted combined hysterectomy: a new surgical concept compared to the classical laparoscopicassisted vaginal hysterectomy by a prospective study. Gynecol Obstet Invest 2017:82:223-229.

61 Ghezzi F, Cromi A, Siesto G, et al. Minilaparoscopic versus conventional laparoscopic hysterectomy: results of a randomized trial. J Minim Invasive Gynecol 2011:18:455-61.

62 Acton JN, Salfinger SG, Tan J, et al. Outcomes of total laparoscopic hysterectomy using a 5-mm versus 10-mm laparoscope: a randomized control trial. J Minim Invasive Gynecol 2016;23:101-6.

63 Shen CC, Wu MP, Lu CH, et al. Effects of closed suction drainage in reducing pain after laparoscopic-assisted vaginal hysterectomy. J Am Assoc Gynecol Laparosc 2003;10:210-4

64 Bogani G, Uccella S, Cromi A, et al. Low vs standard pneumoperitoneum pressure during laparoscopic hysterectomy: prospective randomized trial. J Minim Invasive Gynecol 2014:21:466-71

65 Madsen MV, Istre 0, Staehr-Rye AK, et al. Postoperative shoulder pain after laparoscopic hysterectomy with deep neuromuscular blockade and low-pressure pneumoperitoneum. Eur J Anaesthesiol 2016;33:341-7.

66 Herrmann A, De Wilde RL. Insufflation with humidified and heated carbon dioxide in short-term laparoscopy: a double-blinded randomized controlled trial. Biomed Res Int 2015;2015:1-11.

67 Radosa JC, Radosa MP, Mavrova R, et al. Five minutes of extended assisted ventilation with an open umbilical trocar valve significantly reduces postoperative abdominal and shoulder pain in patients undergoing laparoscopic hysterectomy. Eur J Obstet Gynecol Reprod Biol 2013;171:122-7.

68 Roy KK, Goyal M, Singla S, et al. A prospective randomised study of total laparoscopic hysterectomy, laparoscopically assisted vaginal hysterectomy and non-descent vagina hysterectomy for the treatment of benign diseases of the uterus. Arch Gynecol Obstet 2011;284:907-12.

69 Fagotti A, Vizzielli G, Fanfani F, et al. Randomized study comparing use of THUNDERBEAT technology vs standard electrosurgery during laparoscopic radical hysterectomy and pelvic lymphadenectomy for gynecologic cancer. J Minim Invasive Gynecol 2014;21:447-53.

70 Rothmund R, Kraemer B, Brucker S, et al. Laparoscopic supracervical hysterectomy using EnSeal vs standard bipolar coagulation technique: randomized controlled trial. $J$ Minim Invasive Gynecol 2013;20:661-6.
71 Martinez V, Beloeil H, Marret E, et al. Non-opioid analgesics in adults after major surgery: systematic review with network meta-analysis of randomized trials. $\mathrm{Br} J$ Anaesth 2017;118:22-31.

72 Wang JJ, ST H, Tzeng Jl, et al. The effect of timing of dexamethasone administration on its efficacy as a prophylactic antiemetic for postoperative nausea and vomiting. Anesth Analg 2000;91:136-9.

73 Zargar-Shoshtari K, Sammour T, Kahokehr A, et al. Randomized clinical trial of the effect of glucocorticoids on peritoneal inflammation and postoperative recovery after colectomy. Br J Surg 2009;96:1253-61.

74 EME B, Schut ME, de Quelerij M. Trends in practice and safety measures of epidural analgesia: report of a national survey. Acta Anaesthesio/ Scand 2018.

75 Barazanchi AWH, MacFater WS, Rahiri J-L, et al. Evidence-based management of pain after laparoscopic cholecystectomy: a prospect review update. $\mathrm{Br} J$ Anaesth 2018; 121:787-803.

76 Pitter MC, Simmonds C, Seshadri-Kreaden U, et al. The impact of different surgical modalities for hysterectomy on satisfaction and patient reported outcomes. Interact J Med Res 2014:3:e11.

77 Donnez O, Donnez J, Dolmans M-M, et al. Low pain score after total laparoscopic hysterectomy and same-day discharge within less than 5 hours: results of a prospective observational study. J Minim Invasive Gynecol 2015;22:1293-9.

78 Dedden SJ, Geomini PMAJ, Huirne JAF, et al. Vaginal and laparoscopic hysterectomy as an outpatient procedure: a systematic review. Eur J Obstet Gynecol Reprod Biol 2017;216:212-23.

79 Wright JD, Ananth CV, Lewin SN, et al. Robotically assisted vs laparoscopic hysterectomy among women with benign gynecologic disease. JAMA 2013:309:689-98.

80 Kehlet H, Wilkinson RC, Fischer HBJ, et al. Prospect: evidence-based, procedurespecific postoperative pain management. Best Pract Res Clin Anaesthesiol 2007;21:149-59

81 Kehlet H, Joshi GP. Systematic reviews and meta-analyses of randomized controlled trials on perioperative outcomes: an urgent need for critical reappraisal. Anesth Analg 2015;121:1104-7.

82 Farrar JT, Portenoy RK, Berlin JA, et al. Defining the clinically important difference in pain Outcome measures. Pain 2000;88:287-94.

83 Emshoff R, Bertram S, Emshoff I. Clinically important difference thresholds of the visual analog scale: a conceptual model for identifying meaningful Intraindividual changes for pain intensity. Pain 2011;152:2277-82.

84 Terkawi AS, Mavridis D, Sessler DI, et al. Pain management modalities after total knee arthroplasty: a network meta-analysis of 170 randomized controlled trials. Anesthesiology 2017;126:923-37.

85 Zeballos JL, Lirk P, Rathmell JP. Low-dose ketamine for acute pain management: a timely nudge toward multimodal analgesia. Reg Anesth Pain Med 2018;43:453-5.

86 Hilliard PE, Waljee J, Moser S, et al. Prevalence of preoperative opioid use and characteristics associated with opioid use among patients presenting for surgery. JAMA Surg 2018;153:929. 